Peter Løchte Jørgensen

Lognormal Approximation of Complex Pathdependent Pension Scheme Payoffs

Finance

Research Group 


\title{
Lognormal Approximation of Complex Path-dependent Pension Scheme Payoffs
}

\author{
Peter Løchte Jørgensen* \\ Finance Research Group \\ Department of Business Studies \\ Aarhus School of Business \\ Fuglesangs Allé 4 \\ DK-8210 Aarhus V \\ DENMARK \\ Phone: + 4589486691 \\ e-mail: plj@asb.dk
}

Current version: November 21, 2006

JEL Classification Codes: G23, C10.

Keywords: Account-based pension schemes, return smoothing, payoff distributions, density approximation, Monte Carlo simulation, Asian options.

\footnotetext{
${ }^{*}$ I am grateful for suggestions and useful comments from an anonymous referee, Pierre Collin-Dufresne, Marc De Ceuster, and from participants at the NHH Skinance Symposium in Hemsedal, Norway, the Workshop on Life Insurance Fair Value at EM Lyon, France, and at the 2005 Deloitte European Risk Management Conference in Antwerp, Belgium. Large parts of this research was carried out while the author was affiliated with the University of Aarhus. Financial support from the Danish Mathematical Finance Network is gratefully acknowledged.
} 


\title{
Lognormal Approximation of Complex Path-dependent Pension Scheme Payoffs
}

\begin{abstract}
This paper analyzes an explicit return smoothing mechanism which has recently been introduced as part of a new type of pension savings contract that has been offered by Danish life insurers. We establish the payoff function implied by the return smoothing mechanism and show that its probabilistic properties are accurately approximated by a suitably adapted lognormal distribution. The quality of the lognormal approximation is explored via a range of simulation based numerical experiments, and we point to several other potential practical applications of the paper's theoretical results.
\end{abstract}




\section{Introduction}

Pension saving schemes come in many forms and variations but throughout much of Europe, and in the US and Japan, a predominant contract form has been the so-called participating or with-profits life insurance policy. In the UK, for example, more than half of all outstanding life insurance policies are of the with-profits type, and in Denmark until very recently virtually all occupational pension schemes have been of this type. The main characteristic of with-profits schemes is that policy holders' contributions are pooled and invested in a mutual fund where the investment return is somehow smoothed over time before it is returned to investors' accounts. The idea of the smoothing procedure is to allow the pension plan sponsor to credit policyholders' accounts first with a basic (and often guaranteed) policy interest rate and then to top this off with fairly stable annual bonuses obviously depending on the success of the investment strategy.

While the classical with-profits industry has been successful for many years it has been forced to face a series of severe challenges recently. These challenges relate to solvency problems caused by carelessly issued guarantees, consequences of negligent risk management, and the merciless revelations of new fair value based financial reporting standards, to name a few. The life insurance and pensions (L\&P) industry has also had to face increasing criticism from many sides for the lack of transparency of their traditional with-profits policies. The with-profits policies are not well-defined contracts in the sense that policy holders' ownership of assets, the associated return smoothing mechanism, and the right to receive bonus are not explicitly known. Although the companies' bonus policy can sometimes be fairly accurately estimated and forecasted (see Grosen and Jørgensen (2002)), the opacity and lack of formality in these policies obviously create problems - particularly for outside observers - in relation to for example risk assessment and the fair valuation of liabilities. ${ }^{1}$ There are also clear indications that pension savers are increasingly frustrated over the terms of with-profits contracts and that many shop for more transparent alternatives - mainly the more individualized unit linked products. In many respects, unit linked contracts are quite the opposite of traditional with-profits policies. Unit linked contracts are individual rather than collective. Assets are placed in separate, customer-controlled accounts rather than in professionally managed pools, so clients know the return generating mechanism exactly and there are no inter-generational transfers. Furthermore, features such as rate of return

\footnotetext{
${ }^{1}$ In his analysis of traditional with-profits pension products Brennan (1993) is also forced to conclude that "Since the bonus process is discretionary, there are no formal rules, and this impedes a definitive assessment of its implications."
} 
guarantees are not automatically issued with unit linked policies, but can sometimes be purchased at an additional fee. “New money” - i.e. contributions from new policies - presently pours into unit linked contracts on a worldwide basis. In Denmark for example, unit linked savings products were virtually unknown prior to 2000, but now they account for about one-third of all new L\&P policies.

The problems with the design of traditional with-profits policies that have become apparent in recent years have inspired innovation and creativity in the R\&D departments of L\&P companies, and many new scheme types have been introduced in the new millennium. The new life savings products that are now offered reflect the fact that today's markets have other preferences and requirements than just a few years ago. The new products are often more transparent and the element of collectivity is less than in traditional with-profits policies. Moreover, the once standard embedded guarantees have now typically been removed. However, a certain resistance towards abandoning all of the features of traditional with-profits products is also observed. This holds particularly in relation to the profit smoothing principle which is clearly seen as such a deeplyrooted virtue of traditional L\&P products that L\&P companies have had a hard time letting it go entirely. In this connection it has been argued that one should not throw out the baby with the bath water, as the old saying has it.

This paper analyzes the construction and properties of a representative example of these new pension saving schemes. The most interesting and distinctive feature of this new scheme is that in contrast to traditional with-profits policies, its bonus crediting policy has been made explicit and put on a mathematical formula. ${ }^{2}$ The purpose of this product design has been to alleviate some of the above-mentioned problems related to traditional with-profits policies and to combine the "best of two worlds": the transparency and individuality of the unit linked schemes, and the seemingly more moderate risk structure of traditional return smoothing products.

One obvious advantage of explicit and well-defined return smoothing mechanisms from a researcher's point of view is that with-profits contracts can now be analyzed with the same degree of precision as is obtained in the vast literature on unit-linked policies. The lack of a

\footnotetext{
${ }^{2}$ The pension scheme studied in this paper was marketed by Danish Life Insurer Codan in September 2002. Codan's new pension scheme was named TidsPension (TimePension) and was the first in Denmark to offer a fully transparent and mathematically well-specified return smoothing mechanism. TidsPension quickly became a success for Codan. The launch of the new product created a lot of media attention and Codan - Denmark's 4th biggest life insurer/pension provider - now sells more TidsPension policies than unit linked policies. Moreover, Codan has almost stopped selling traditional with-profits policies. Further background, discussion, and analysis is provided in Guillen, Jørgensen, and Nielsen (2006).
} 
similar body of literature on with-profits products is undoubtedly attributable to the previous lack of formalism in the definition of these contracts. We can thus focus more precisely on the true risk properties and other probabilistic characteristics of these contracts and these are some of the subjects that will be analyzed in this paper.

The remainder of the paper is organized as follows. The next section describes the design characteristics of the contract to be analyzed. The payoff function implied by the return smoothing mechanism is established, and a dynamic model for the financial market and the investment fund underlying the contract is introduced. Section 3 contains our main theoretical results. We argue that the complex and path-dependent payoff function of the contract may be well approximated by a lognormal distribution. By establishing the true first and second moments of the payoff function, the moment matching approximating lognormal distribution is then identified. In section 4 the quality of the suggested approximation technique is evaluated by considering a range of realistic examples. These representative examples indicate that the approximation is generally very accurate. This analysis also inspires the definition of a smoothing index which measures the effectiveness of the return smoothing mechanism in removing volatility from the underlying investment fund. Section 5 concludes and discusses other potential practical applications of the paper's results.

\section{The contract and the model}

This section first describes the design of the contract to be studied. We then derive the contract's payoff function and introduce a simple model for the market dynamics which will allow us to analyze the probabilistic properties of the payoff function.

\subsection{Contract design}

For notational simplicity we study the single premium version of the contract. ${ }^{3}$ The contract is entered into at time 0 , and it has a fixed maturity date, $T$, and a number, $N$, of smoothing dates, $t_{n}, n=1, \ldots, N$, that are equally spaced over the life span of the contract as illustrated by the figure below. ${ }^{4}$

\footnotetext{
${ }^{3}$ In practice both single and periodic premium contracts are offered. Periodic premium contracts are clearly the more common choice of pension savers.

${ }^{4}$ In the real life version of this contract $\Delta t=\frac{T}{N}$ is equal to 1 month.
} 


\section{Figure 1: Time structure of the model}

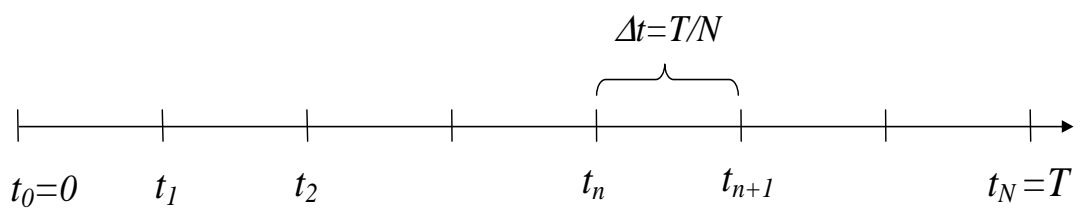

The central point of the contract is the policyholder's account into which his single premium is deposited at time 0 . The balance in this account will be updated only at the smoothing dates and finally paid out at the maturity date, $T$. The policyholder's account balance is not payable before time $T$. The rule for updating the policyholder's account balance at the smoothing dates is also referred to as the return smoothing mechanism and is described next.

When the pension company receives the initial premium it is immediately invested in a wellspecified financial portfolio. For $t \in[0, T]$ let $A(t)$ denote the time $t$ (cum-dividend) market value of this underlying investment fund. $\{A(t)\}_{0 \leq t \leq T}$ is a continuous time process and the idea of the return smoothing mechanism is to let the development in the policyholder's account balance "shadow" the development in the value of the investment fund in such a way that large fluctuations in market values are smoothed out over time.

With $D(t)$ denoting the policyholder's account balance at time $t \in[0, T]$, the return smoothing mechanism is defined in the following way:

$D(t)= \begin{cases}D(0)=A(0) & t \in\left[t_{0}, t_{1}[\right. \\ \left(1+r_{D}\right) D\left(t_{n-1}\right)+\alpha\left(A\left(t_{n}\right)-\left(1+r_{D}\right) D\left(t_{n-1}\right)\right) & t \in\left[t_{n}, t_{n+1}[, n \in\{1, \ldots, N\} .\right.\end{cases}$

Here $r_{D}$ is the constant and positive discretely compounded reference policy interest rate, and $\alpha \in[0,1]$ is the smoothing parameter. ${ }^{5}$

We note first that $\{D(t)\}_{0 \leq t \leq T}$ is a pure jump-process in continuous time with jumps - i.e. account balance updates - occuring at the smoothing dates. It is then observed that the simple

\footnotetext{
${ }^{5}$ Both $r_{D}$ and $\alpha$ are expressed on a periodic basis. The relations to the corresponding annualized rates, $r_{D}^{a n n}$ and $\alpha^{a n n}$, are given as $1+r_{D}=\left(1+r_{D}^{a n n}\right)^{\Delta t}$ and $1-\alpha=\left(1-\alpha^{a n n}\right)^{\Delta t}$ respectively.
} 
modus operandi of the return smoothing mechanism is as follows: When a smoothing date is reached, the policyholder's account balance is first credited with the fixed reference policy interest rate. Then the market value of the underlying investment fund, $A\left(t_{n}\right)$, is sampled and the surplus (or deficit) in the interim buffer, $A\left(t_{n}\right)-\left(1+r_{D}\right) D\left(t_{n-1}\right)$, is determined. A fraction, $\alpha$, of this surplus is then further credited to the policyholder's account as a kind of "bonus". It should be noted that as the surplus may well become negative, so can the "bonus". Note finally that the lower the $\alpha$, the more the market returns on $A(\cdot)$ are smoothed towards the certain "promised" reference return, $r_{D}$. We can thus refer to the limiting cases as full smoothing $(\alpha=0)$ and no smoothing $(\alpha=1)$ respectively.

To illustrate the properties of the smoothing mechanism we provide the following simple 5-period example where the underlying investment fund experiences the return series $+20 \%$, $-15 \%,+20 \%,-15 \%,+20 \%$ over the 5 periods. The reference policy interest rate, $r_{D}$, is $3 \%$, the smoothing parameter, $\alpha$, is $20 \%$, and the contract size is 100 . In this representative scenario the value of the investment fund will be 120 after the first period. According to (1) the policyholder's new account balance at time 1 is determined as $D(1)=1.03 \cdot 100+0.2(120-1.03 \cdot 100)=106.4$. Values and account balances for the full period are shown in Table 1 below and the development is further illustrated in Figure 2.

\section{Table 1: Smoothing of market returns: An example}

\begin{tabular}{|ccc|}
\hline \hline $\begin{array}{c}\text { Numerical example of the development } \\
\text { in the investment fund value and } \\
\text { the policyholder account balance }\end{array}$ \\
$N=T=5, \Delta t=1, r_{D}=0.03, \alpha=0.2$ \\
\hline Time & Fund value & Account balance \\
$t$ & $A(t)$ & $D(t)$ \\
\hline 0 & 100.00 & 100.00 \\
1 & 120.00 & 106.40 \\
2 & 102.00 & 108.07 \\
3 & 122.40 & 113.53 \\
4 & 104.04 & 114.36 \\
5 & 124.85 & 119.20 \\
\hline \hline
\end{tabular}




\section{Figure 2: Smoothing of market returns: An example}

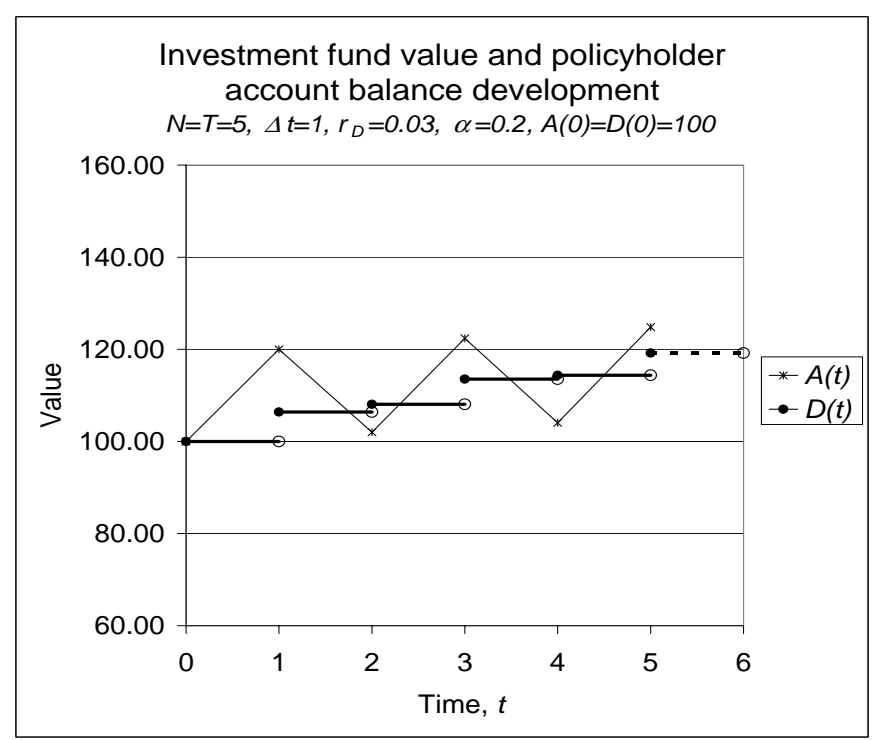

Figure 2 clearly illustrates how the balance in the policyholder's account, $D(t)$, tracks the market value of the investment fund, $A(t)$, while at the same time being the less volatile of the two processes. In the present scenario the issuer of the contract ends up with a surplus since $A(5)>D(5)$. In other scenarios the insurer might experience a deficit at the maturity date.

\subsection{The payoff function and the dynamic model}

As explained above, the contract's payoff at time $T$ is $D(T)$, and to obtain a more explicit representation of this payoff function we can susbstitute $D(\cdot)$ 's recursively in (1) to first find

$$
D(T)=(1-\alpha)^{N-n}\left(1+r_{D}\right)^{N-n} D\left(t_{n}\right)+\alpha \sum_{i=n+1}^{N}(1-\alpha)^{N-i}\left(1+r_{D}\right)^{N-i} A\left(t_{i}\right) .
$$

Taking the recursive substitutions all the way back to time zero we arrive at

$$
D(T)=(1-\alpha)^{N}\left(1+r_{D}\right)^{N} D(0)+\alpha \sum_{i=1}^{N}(1-\alpha)^{N-i}\left(1+r_{D}\right)^{N-i} A\left(t_{i}\right) .
$$

Relation (3) shows that the contract's final payoff can be decomposed into a known constant - a "bond element" - and an exponentially weighted sum of fund values sampled at the smoothing dates - a kind of "structured derivative". It should be noted that important information about $D(T)$ is thus revealed as time passes and as the $A\left(t_{i}\right) \mathrm{s}$ are realized. For $t \in\left[t_{n}, t_{n+1}[\right.$, for 
example, decomposition (2) clarifies how $D\left(t_{n}\right)$ - which is then observed - captures relevant information about previous $A\left(t_{i}\right)$ s.

In order to study the properties of the contract further it is now time to make specific assumptions about the financial market and the dynamics of the underlying investment fund. Let us therefore assume that all activity occurs on a filtered probability space $\left(\Omega, \mathcal{F},\{\mathcal{F}\}_{t}, \mathcal{P}\right)$ supporting Brownian motion on the finite time interval $[0, T]$. The value of the investment fund evolves through time according to a geometric Brownian motion, i.e.

$$
d A(t)=\mu A(t) d t+\sigma A(t) d \mathcal{W}(t), \quad A(0)=D(0)
$$

where $\mu$ and $\sigma$ are the positive and constant drift and diffusion coefficients respectively. $\mathcal{W}(t)$ is a standard Brownian motion under the (physical) probability measure $\mathcal{P}$. Adding the assumptions of existence of perfect markets and of a constant continuously compounded riskless rate of interest, $r$, our framework would thus be identical to the celebrated framework of Black and Scholes (1973). The well-known solution to (4) is

$$
A(u)=A(t) e^{\left(\mu-\frac{1}{2} \sigma^{2}\right)(u-t)+\sigma(\mathcal{W}(u)-\mathcal{W}(t))}, \quad 0 \leq t \leq u \leq T
$$

which implies that future fund values are lognormally distributed.

For the remaining part of the paper our main objects of interest will be the stochastic "structured derivative” components of (2) and (3), and for $t \in\left[t_{n}, t_{n+1}\right.$ [ we therefore define

$$
X_{n, N}=\alpha \sum_{i=n+1}^{N}(1-\alpha)^{N-i}\left(1+r_{D}\right)^{N-i} A\left(t_{i}\right) .
$$

With (5) as the model for the dynamics of the underlying investment fund, $X_{n, N}$ is clearly a stochastic variable constructed as an exponentially weighted sum of correlated lognormal variables. Such (weighted) sums of correlated lognormal variables frequently arise in finance and insurance contexts, and it is therefore unfortunate that the density function of the sum of lognormal variables in general does not have an explicit representation. Various approximation techniques have therefore been developed and explored. One common and popular methodology has been to approximate the sum of lognormals by a suitably adapted (moment matching) lognormal distribution. Dufresne (2004) provides theoretical justification for this approach and for example shows that the lognormal is the limit distribution as the volatility tends to zero. In an earlier paper Dufresne (1990) proves that the infinite sum of lognormals has a reciprocal gamma 
distribution. This result provides motivation for the potential use of the moment matching reciprocal gamma distribution as an alternative for approximating the distribution of a finite sum of lognormal variables. Finally, comonotonic approximations have recently been proposed as yet another interesting approximation technique when working with discrete sums of lognormal variables, see e.g. Dhaene et al. (2002a).

The above-mentioned approximation techniques have all been applied to price Asian options a problem that has been studied extensively in the financial literature. ${ }^{6}$ For example, Levy (1992) provides approximation formulas for Asian option values based on the moment matching lognormal approximation. ${ }^{7}$ Milevsky and Posner (1998) base their analysis of Asian options on the aforementioned approximating reciprocal gamma distribution, and Dhaene et al. (2002b) derive comonotonic upper and lower bounds for Asian option prices which appear to be very tight.

The cited works on Asian option pricing are of obvious relevance for the problem studied in this paper because of the similarity of the pension contract's payoff function given in (6) and the variable underlying the exotic path-dependent structured derivatives that we know as Asian options. However, having noted some very qualified alternative procedures for approximating sums of lognormal variables, we henceforth confine ourselves to working exclusively with the moment matching lognormal approximation. We thus leave it for future research to further compare and evaluate the competing methodologies for approximating sums of lognormal variables.

The paper's next section will address the specifics in determining the approximating lognormal density functions for the family of $X_{n, N}$ s.

\section{Approximating the contract's payoff distribution}

As noted in the previous section it is well-known that the distributions of sums of lognormal variables may often be well approximated by suitably adapted lognormal distributions. We suspect that a similar approach can be used in the present context, and pursue the task of finding appropriate approximating lognormal densities for $X_{n, N}$ in this section. The critieria for selecting "suitable" approximating lognormal distributions will be that of matching moments as explained below.

\footnotetext{
${ }^{6}$ Asian options are options where the payoff depends on the average price of the underlying asset during at least some part of the life of the option. Prices are lognormal when the typical Black and Scholes (1973) assumptions are made.

${ }^{7}$ In parts of their study on American-style Asian options Hansen and Jørgensen (2000) pursue a similar idea.
} 
Let first $\hat{X}_{n, N} \mid \mathcal{F}_{t}$ denote the lognormal random variable the (conditional) density of which we will use to approximate the density of $X_{n, N} \mid \mathcal{F}_{t}$. The variable $\ln \hat{X}_{n, N} \mid \mathcal{F}_{t}$ is then normally distributed with mean and variance which we denote $\xi_{t}$ and $\nu_{t}^{2}$ respectively. The moment generating function of the normal tells us that

$$
E\left\{\hat{X}_{n, N}^{k} \mid \mathcal{F}_{t}\right\}=\exp \left\{k \xi_{t}+\frac{1}{2} k^{2} \nu_{t}^{2}\right\}, \quad k \in \mathbb{R} .
$$

In particular, the first two moments $(k=1$ and $k=2)$ are given as

$$
\begin{aligned}
& E\left\{\hat{X}_{n, N} \mid \mathcal{F}_{t}\right\}=\exp \left\{\xi_{t}+\frac{1}{2} \nu_{t}^{2}\right\} \\
& E\left\{\hat{X}_{n, N}^{2} \mid \mathcal{F}_{t}\right\}=\exp \left\{2\left(\xi_{t}+\nu_{t}^{2}\right)\right\}
\end{aligned}
$$

Now, as will be established in the lemmas below, the true moments $E\left\{X_{n, N} \mid \mathcal{F}_{t}\right\}$ and $E\left\{X_{n, N}^{2} \mid \mathcal{F}_{t}\right\}$ can be explicitly calculated, and we will therefore use the following moment matching conditions to obtain $\xi_{t}$ and $\nu_{t}^{2}$ to use in the approximating density:

$$
\begin{aligned}
& E\left\{\hat{X}_{n, N} \mid \mathcal{F}_{t}\right\}=E\left\{X_{n, N} \mid \mathcal{F}_{t}\right\} \\
& E\left\{\hat{X}_{n, N}^{2} \mid \mathcal{F}_{t}\right\}=E\left\{X_{n, N}^{2} \mid \mathcal{F}_{t}\right\}
\end{aligned}
$$

Using (8) and (9), the system (10)-(11) can be solved for $\xi_{t}$ and $\nu_{t}^{2}$. We get

$$
\begin{aligned}
\xi_{t} & =2 \ln E\left\{X_{n, N} \mid \mathcal{F}_{t}\right\}-\frac{1}{2} \ln E\left\{X_{n, N}^{2} \mid \mathcal{F}_{t}\right\}, \\
\nu_{t}^{2} & =\ln E\left\{X_{n, N}^{2} \mid \mathcal{F}_{t}\right\}-2 \ln E\left\{X_{n, N} \mid \mathcal{F}_{t}\right\} .
\end{aligned}
$$

The matching of the first two moments in this way has been termed Wilkinson's approximation (see e.g. Levy (1992)), and with the right-hand sides of (12) and (13) known, this will be our recipe for selecting approximating lognormal distributions. The lognormal density is of course given as

$$
f\left(x ; \xi_{t}, \nu_{t}\right)=\frac{1}{\sqrt{2 \pi \nu_{t}^{2}} x} \exp \left\{-\frac{1}{2}\left(\frac{\ln x-\xi_{t}}{\nu_{t}}\right)^{2}\right\}, \quad x>0 .
$$

The following lemmas establish formulas for the true moments to be used on the right-hand sides of (12) and (13). 
Lemma 1: Let $t \in\left[t_{n}, t_{n+1}[\right.$ with $n \in\{0,1, \ldots, N-1\}$. We have

$$
E\left\{X_{n, N} \mid \mathcal{F}_{t}\right\}=\alpha A(t) e^{\mu(T-t)} \cdot \frac{1-\frac{(1-\alpha)^{N-n}\left(1+r_{D}\right)^{N-n}}{e^{\mu\left(T-t_{n}\right)}}}{1-\frac{(1-\alpha)\left(1+r_{D}\right)}{e^{\mu \Delta t}}}
$$

\section{Proof of Lemma 1: See Appendix A.}

Remarks: We note the following properties of the expression for the conditional expectation established in (15):

1. $E\left\{X_{n, N} \mid \mathcal{F}_{t}\right\}$ does not depend on the investment fund volatility, $\sigma$.

2. $E\left\{X_{n, N} \mid \mathcal{F}_{t}\right\}=0$ for $\alpha=0$. This makes sense since with $\alpha=0$ (full smoothing) the stochastic component of the payoff function disappears and the contract is equivalent to a riskless bond.

3. $E\left\{X_{n, N} \mid \mathcal{F}_{t}\right\}=A(t) e^{\mu(T-t)}$ for $\alpha=1$. This is because with $\alpha=1$ (no smoothing), $X_{n, N} \mid \mathcal{F}_{t}$ is identical to $A(T) \mid \mathcal{F}_{t}$ and therefore lognormal. In this case holding the contract is thus precisely like holding the investment fund for which the same result holds, i.e. $E\left\{A(T) \mid \mathcal{F}_{t}\right\}=A(t) e^{\mu(T-t)}$.

4. Note finally that in the special case where $(1-\alpha)\left(1+r_{D}\right)=1$ (which corresponds to equal weighting in the sum (6)) we have shown that

$$
E\left\{\sum_{i=n+1}^{N} A\left(t_{i}\right) \mid \mathcal{F}_{t}\right\}=A(t) e^{\mu(T-t)} \frac{1-e^{-\mu\left(T-t_{n}\right)}}{1-e^{-\mu \Delta t}} .
$$

This result corresponds with the result obtained by Levy (1992), p. 489, in his study on Asian options, i.e. the equal weighting case.

Figure 3 below shows representative plots of the expectation of the two contract payoff components (and their sum) as functions of the (annual) smoothing parameter, $\alpha^{a n n} .8$ The remaining

\footnotetext{
${ }^{8}$ See footnote 3 for the relation between the periodic smoothing parameter, $\alpha$, used throughout the paper in the formulas, and the annualized smoothing parameter, $\alpha^{a n n}$.
} 
parameters are fixed at $T=5, A(0)=D(0)=100, \Delta t=\frac{1}{12}, r_{D}^{a n n}=0.03$, and $\mu=0.07$. From (3) the first payoff component - the "bond element" - is a deterministic and decreasing (power) function of $\alpha$. The plot shows that this component dominates for small values of $\alpha$, and that it decreases quickly in $\alpha$ so that for example for $\alpha^{a n n}=0.2$ the bond element only accounts for about $30 \%$ of the expected total payoff.

The expectation of the stochastic part of (3) is plotted using the result in Lemma 1. It is seen that $E\left\{X_{n, N} \mid \mathcal{F}_{t}\right\}$ increases in $\alpha$. This can be explained by the fact that in the present case a higher $\alpha$ corresponds to less smoothing and thus to taking on more risk. The sum of the parts is also increasing in $\alpha$ in this case, but both of these relationships might be reversed for example by the (unnatural) choice of $r_{D}^{a n n}$ such that $r_{D}^{a n n}>\mu$.

\section{Figure 3}

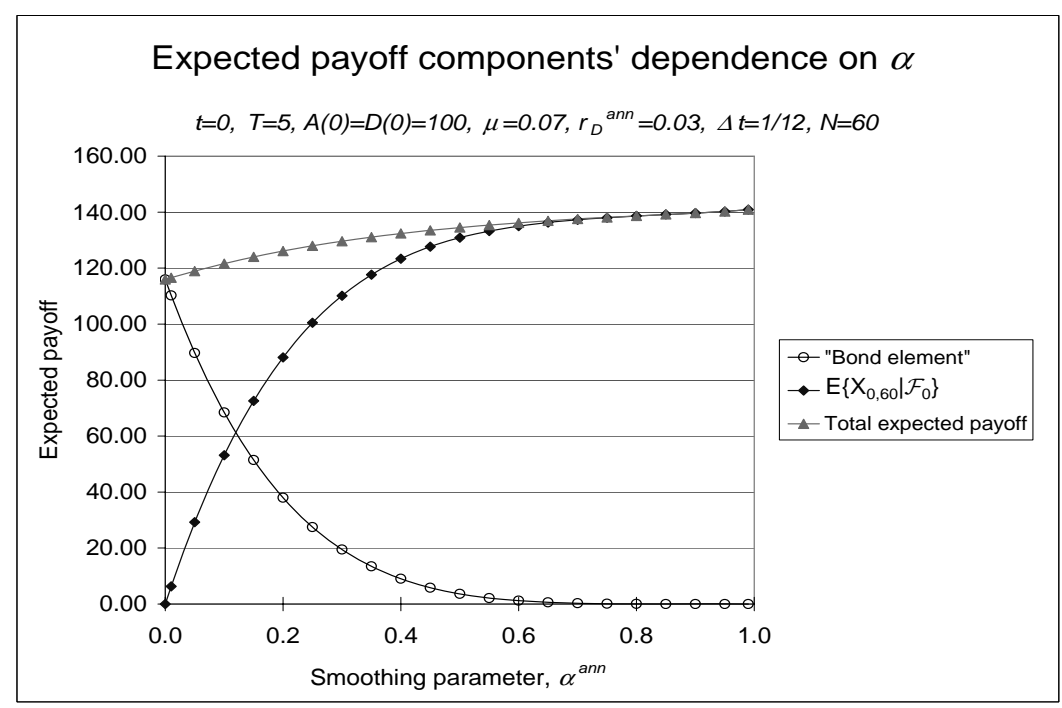

We move on to establishing the second moment of $X_{n, N}$ in Lemma 2 below. 
Lemma 2: Let again $t \in\left[t_{n}, t_{n+1}[\right.$ with $n \in\{0,1, \ldots, N-1\}$. As regards the second conditional moment of $X_{n, N}$, the following result can be established:

$$
E\left\{X_{n, N}^{2} \mid \mathcal{F}_{t}\right\}=\alpha^{2} A(t)^{2} e^{\left(2 \mu+\sigma^{2}\right)(T-t)}\left[\Psi_{1}-\Psi_{2}+\Psi_{3}-\Psi_{4}\right]
$$

where

$$
\begin{aligned}
\Psi_{1} & =\left(\frac{\Lambda^{N-n}}{\Lambda-1}\right)\left(\frac{\Gamma^{N-n}-1}{\Gamma-1}\right) \\
\Psi_{2} & =\left(\frac{\Lambda}{\Lambda-1}\right)\left(\frac{(\Gamma \Lambda)^{N-n}-1}{\Gamma \Lambda-1}\right) \\
\Psi_{3} & =\left(\frac{\Gamma}{\Gamma-1}\right)\left(\frac{\left.(\Gamma \Lambda)^{N-n}-1\right)}{\Gamma \Lambda-1}\right) \\
\Psi_{4} & =\left(\frac{1}{\Gamma-1}\right)\left(\frac{\Lambda^{N-n}-1}{\Lambda-1}\right) \\
\Gamma & =\frac{(1-\alpha)\left(1+r_{D}\right)}{e^{\mu \Delta t}} \\
\Lambda & =\frac{(1-\alpha)\left(1+r_{D}\right)}{e^{\left(\mu+\sigma^{2}\right) \Delta t}} .
\end{aligned}
$$

Proof of Lemma 2: See Appendix B.

Remarks: The following observations can be made in relation to the second conditional moment derived in (16):

1. $E\left\{X_{n, N}^{2} \mid \mathcal{F}_{t}\right\}$ clearly depends on the investment fund volatility, $\sigma$.

2. Letting $\sigma=0$ it is easily seen that $E\left\{X_{n, N}^{2} \mid \mathcal{F}_{t}\right\}=E\left\{X_{n, N} \mid \mathcal{F}_{t}\right\}^{2}$ implying that $\operatorname{Var}\left\{X_{n, N} \mid \mathcal{F}_{t}\right\}=$ 0 - an intuitively appealing result.

3. $E\left\{X_{n, N}^{2} \mid \mathcal{F}_{t}\right\}=0$ for $\alpha=0$. Again this is explained by the fact that the entire stochastic term of the payoff function - given in (2) or (3) - disappears when $\alpha=0$.

4. For $\alpha=1$ we get $E\left\{X_{n, N}^{2} \mid \mathcal{F}_{t}\right\}=A(t)^{2} e^{\left(2 \mu+\sigma^{2}\right)(T-t)}$. This is consistent with the lognormality of $X_{n, N}(=A(T))$ when $\alpha=1$. Recall that $\ln A(T) \mid \mathcal{F}_{t} \sim \mathcal{N}\left(\left(\mu-\frac{1}{2} \sigma^{2}\right)(T-\right.$ $\left.t), \sigma^{2}(T-t)\right)$. The moment generating function, (7), can be used to confirm the result. 
5. In the special case where $(1-\alpha)\left(1+r_{D}\right)=1$, it can be confirmed that (16) simplifies to the result obtained by Levy (1992) (see p. 489 ibid.).

For the same representative case as used in Figure 3 above, Figure 4 shows how the second moment of the payoff function varies with the smoothing parameter, $\alpha^{a n n}$, and with the fund volatility, $\sigma$. As expected we see that $E\left\{X_{n, N}^{2} \mid \mathcal{F}_{t}\right\}$ increases in $\alpha^{a n n}$ as well as in $\sigma$. Again these relations are robust for $\mu>r_{D}^{a n n}$, but might be reversed for the (rather unnatural) cases where $r_{D}^{a n n}>\mu$.

\section{Figure 4}

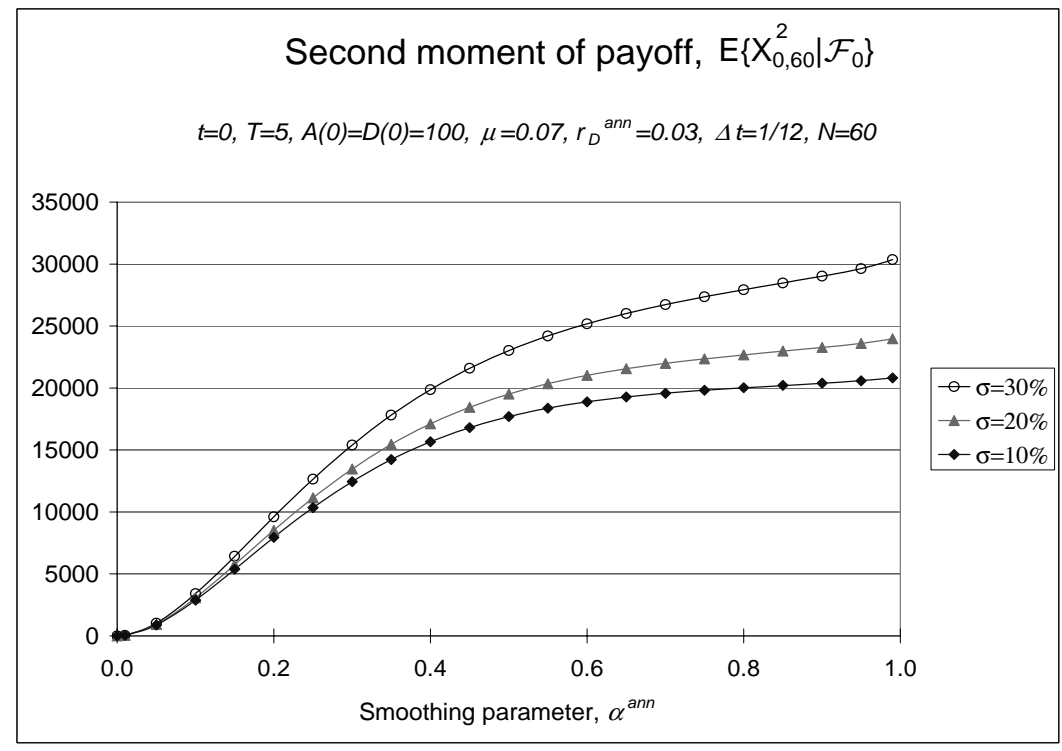




\section{Evaluation of the approximation method}

\subsection{Approximation of unconditional payoff densities}

To evaluate the quality of the suggested approximating lognormal density for $X_{n, N}$ we provide a variety of results comparing the approximations with the "true" densities obtained via largescale Monte Carlo simulation. We first concentrate on unconditional densities for the contract payoff, $D(T)$, and "stress test" the approximation methodology by considering the quality of the approximation for a collection of rather extreme cases which are described next.

We consider the 8 borderline cases that arise as combinations of situations where time to maturity is short or long, where volatility is low or high, and where smoothing is weak or strong. More specifically we consider the cases identified and listed in Table 2 below:

Table 2

\begin{tabular}{|c|c|c|c|c|c|c|}
\hline \multicolumn{7}{|c|}{$\begin{array}{l}\text { Overview of cases for comparison and evaluation of } \\
\text { the quality of the lognormal approximation of } X_{n, N}\end{array}$} \\
\hline Case & Time to Maturity & Volatility & Smoothing & $T$ & $\sigma$ & $\alpha^{a n n}$ \\
\hline 1 & Short & Low & Strong & 5 & $10 \%$ & $5 \%$ \\
\hline 2 & Short & Low & Weak & 5 & $10 \%$ & $20 \%$ \\
\hline 3 & Short & High & Strong & 5 & $30 \%$ & $5 \%$ \\
\hline 4 & Short & High & Weak & 5 & $30 \%$ & $20 \%$ \\
\hline 5 & Long & Low & Strong & 20 & $10 \%$ & $5 \%$ \\
\hline 6 & Long & Low & Weak & 20 & $10 \%$ & $20 \%$ \\
\hline 7 & Long & High & Strong & 20 & $30 \%$ & $5 \%$ \\
\hline 8 & Long & High & Weak & 20 & $30 \%$ & $20 \%$ \\
\hline \multicolumn{7}{|c|}{ Other, fixed parameters: } \\
\hline
\end{tabular}

The choice of $10 \%$ and $30 \%$ as borderline volatilities has been motivated by the fact that welldiversified portfolios as well as broad stock exchange indices typically have volatilities in the neighborhood of $15-20 \%$. Further, a time to maturity of 5 years must be considered as short in a pensions savings context, whereas anything above 20 years would qualify for the "long" predicate. As regards the smoothing parameter it will become clearer later in the paper that an 
annual $\alpha$ of $20 \%$ or above does not imply a lot of smoothing. Hence we have taken $\alpha^{a n n}=5 \%$ and $\alpha^{a n n}=20 \%$ to represent "strong" and "weak" smoothing respectively.

The remaining parameter values are fixed and identical for the 8 cases considered. We set $\mu=7 \%, r_{D}^{a n n}=3 \%$, and use monthly smoothing, i.e. $\Delta t=\frac{1}{12}$. In each case we simulate $1,000,000$ paths for the value of the underlying investment fund to obtain 1,000,000 final contract payoffs, $D(T)$. This allows for the construction of very accurate "true" density and distribution functions for $D(T)$. In the following we will compare these with approximated densities and distributions obtained from using the methodology described in the previous section. We obtain approximate distributions for $D(T)$ by displacing in each case the distribution for $X_{0, N}$ by the constant - "the bond element" - given by the first term in (3).

The results of this effort can be studied in the appended Figures 5-12. Each figure consists of three plots. The first plot of each figure shows the simulated true density together with the corresponding (displaced) approximating lognormal density. Ideally, these density functions should coincide. The second plots are density difference plots with the true simulated density subtracted from the lognormal approximation. Ideally, all differences should be zero and the plotted curve should coincide with the $x$-axis. Finally, $Q-Q$ plots (quantiles of simulated against quantiles of approximate distribution) are provided as the third plot of the figures. The perfect $Q-Q$ plot is, of course, a straight $45^{\circ}$-line.

Insert Figures 5-12 about here.

The general impression from considering the plots in Figures 5-12 is that the lognormal approximation method works very well. In most of the cases it is hard to distinguish the plot of the approximating density from the plot of the true density. Where small differences occur these tend to be for cases where volatility is high and/or smoothing is strong. The differences can be further explored in the density difference plots. These plots are seen to display a similar wavy pattern across the eight cases: The lognormal approximation first overshoots a little, then undershoots, overshoots, and finally undershoots again. Note, however, the very small (and generally somewhat different) $y$-axis scales in these plots.

The $Q$ - $Q$-plots support the conclusions above. They are all close to being perfect $45^{\circ}$-lines. Case 7 which has both high volatility, strong smoothing, and a long time to maturity seems to be the "worst of the good". 


\subsection{Digressions on replication and on measuring the extent of smoothing}

If we accept the conclusion that the part of the payoff function represented by $X_{n, N}$ is well approximated by a lognormal distribution, then we must also conclude that a payoff structure almost identical to the one provided by the return smoothing contract can be obtained by investing partly in a riskless asset and partly directly in an asset, $S$, whose price process is a geometric Brownian motion (GBM) with an appropriately scaled-down volatility. ${ }^{9}$ If $S$ evolves as a GBM with volatility $\sigma_{S}$ we have

$$
\operatorname{Var}\left(\ln S_{T} \mid \mathcal{F}_{t}\right)=\sigma_{S}^{2}(T-t)
$$

and since for $t \in\left[t_{n}, t_{n+1}[\right.$ we have

$$
\operatorname{Var}\left(\ln \hat{X}_{n, N} \mid \mathcal{F}_{t}\right)=\nu_{t}^{2}
$$

then at any time $t$ a good substitute (read: "hedge”) for the second, stochastic payoff component, (6), of the return smoothing mechanism would be a direct investment in asset $S$ with a volatility given as

$$
\sigma_{S}=\frac{\nu_{t}}{\sqrt{T-t}}
$$

This volatility is easily determined using the definition of $\nu_{t}$ given in (13) which can be evaluated using the expressions derived in Lemmas 1 and 2. It should be noted that $\sigma_{S}$ changes with time.

One might finally expand the considerations above to develop a measure of the strength of smoothing implied by a specific set of contract parameters at a given point in time. In order to make sense, such a measure should use the volatility of the underlying investment fund as the base for comparison, and it should express how much of this volatility is "removed" by imposing the return smoothing mechanism on this fund. To measure the volatility of the contract's smoothed return, relation (19) can serve as the point of departure. Recall that $\sigma_{S}$ is the approximate annualized volatility on the second, stochastic part of the payoff (see (2)). The volatility of the first, riskless part is zero. To obtain an approximate total return volatility, $\sigma_{S}$ should be weighted to reflect the relative importance of $X_{n, N}$ in the payoff function. A quick-and-dirty choice of weight, $\varphi$, is obtained by using the expectations of the two payoff components in the weighting

\footnotetext{
${ }^{9}$ Assuming financial markets to be in no-arbitrage equilibrium we can ignore considerations on the expected return here.
} 
scheme:

$$
\varphi=\frac{E\left\{X_{n, N} \mid \mathcal{F}_{t}\right\}}{E\left\{D(T) \mid \mathcal{F}_{t}\right\}}=\frac{E\left\{X_{n, N} \mid \mathcal{F}_{t}\right\}}{(1-\alpha)^{N-n}\left(1+r_{D}\right)^{N-n} D\left(t_{n}\right)+E\left\{X_{n, N} \mid \mathcal{F}_{t}\right\}}
$$

This is again easily evaluated using the result in Lemma 1.

A sensible measure of the strength of smoothing - a smoothing index - can now be defined as follows:

$$
\text { Smoothing index }=\frac{\sigma-\varphi \sigma_{S}}{\sigma} \cdot 100
$$

Table 3 below displays numerical values of this smoothing measure for representative combinations of various parameter values.

\begin{tabular}{|c|c|c|c|c|c|c|c|c|c|c|}
\hline & \multicolumn{9}{|c|}{$\begin{array}{l}\text { Smoothing index* } \\
=100, r_{D}^{a n n}=0.03, \mu=0.07, \Delta t=\frac{1}{12}\end{array}$} \\
\hline & & \multicolumn{3}{|c|}{$\alpha^{a n n}=0.05$} & \multicolumn{3}{|c|}{$\alpha^{a n n}=0.20$} & \multicolumn{3}{|c|}{$\alpha^{a n n}=0.50$} \\
\hline & & $\sigma=0.1$ & $\sigma=0.2$ & $\sigma=0.3$ & $\sigma=0.1$ & $\sigma=0.2$ & $\sigma=0.3$ & $\sigma=0.1$ & $\sigma=0.2$ & $\sigma=0.3$ \\
\hline \multirow{3}{*}{$T$} & 5 & 84.8 & 84.7 & 84.6 & 52.6 & 52.4 & 52.0 & 21.8 & 21.5 & 21.1 \\
\hline & 10 & 70.4 & 70.0 & 69.5 & 30.9 & 30.3 & 29.4 & 10.5 & 10.3 & 10.0 \\
\hline & 20 & 47.0 & 46.0 & 44.5 & 15.0 & 14.5 & 13.6 & 5.1 & 5.0 & 4.9 \\
\hline
\end{tabular}

\section{Table 3}

* The smoothing index expresses (in percentage terms) how large a proportion of the volatility of the underlying investment fund is removed by imposing the return smoothing mechanism to this fund.

Table 3 confirms that a lower $\alpha^{a n n}$ is generally associated with stronger smoothing. The index suggested in (21) tends to 100 as $\alpha^{\text {ann }} \downarrow 0$, and it will tend to 0 as $\alpha^{a n n} \uparrow 1$. The table also shows that the volatility of the underlying investment fund itself does not affect the smoothing index much. Finally, it is seen that the longer the time to maturity, the weaker the smoothing. Note for example that for $T=20, \sigma=0.20$, and $\alpha=0.20$ - which is a parameter set closely representing what is actually offered in practice - the smoothing index stands only at about 15. This means that the contract volatily is only marginally lower than the volatility of a direct investment in the underlying investment fund. 


\subsection{Approximation of conditional densities}

So far our investigations into the quality of the suggested approximation methodology has centered around examples of unconditional distributions. That is, we have only considered contracts and their payoff distributions as seen from the initial date, $t=0$. However, the approximation procedure was designed to include handling of conditional distributions and approximate payoffs of what might be called in progress contracts at any time $t \in\left[t_{n}, t_{n+1}[, \forall n\right.$. In these situations, the true as well as the approximated payoff distributions will be conditional on observed values of the state variables $D\left(t_{n}\right)$ and $A(t)$ - i.e. the current policyholder account balance and the current market value of the underlying investment fund respectively.

In this section we briefly present some selected illustrative examples to support the claim that the payoff density approximation technique also works well in its conditional form. The examples consider a 20-year contract 15 years after inception and thus with 5 years left to maturity. The initial deposit is normalized at 100 as before, and key contract and market parameters are chosen to reflect a "difficult case" for the approximation technique, cf. the previous section. More specifically, we set $\alpha^{a n n}=0.05$ (strong smoothing) and $\sigma=0.30$ (large volatility). As before we set $\mu=0.07, r_{D}^{a n n}=0.03, \Delta t=\frac{1}{12}$. The remaining determinants of the conditional payoff density are $D(15)$ and $A(15)$. In the present illustration we assume that the policyholder's account has grown precisely according to the expected rate of return on the underlying investment fund. That is, we assume $D(15)=100 \cdot e^{0.07 \cdot 15}=285.77$.

We will then consider three quite different cases for the market value of the underlying fund. Case 1 represents a depressed situation where $A(15)=85.77$. The issuing company thus stands with an accounting deficit of 200. Case 2 is a balanced situation where we let $A(15)=D(15)=285.77$. Finally, Case 3 represents an excellent current market situation where $A(15)=485.77$. The issuing company's account surplus is 200 and prospects for the policyholder are also bright.

Following a similar Monte Carlo procedure as described in the previous section we construct accurate true payoff densities for the 3 cases and in Figure 13 these are plotted together with their associated lognormal approximations.

We first note that the approximation again seems to be very accurate and we therefore leave out the density difference plots as well as the $Q-Q$ plots for these cases. The importance of the current market value of the underlying fund for the final payoff distribution should also be 


\section{Figure 13}

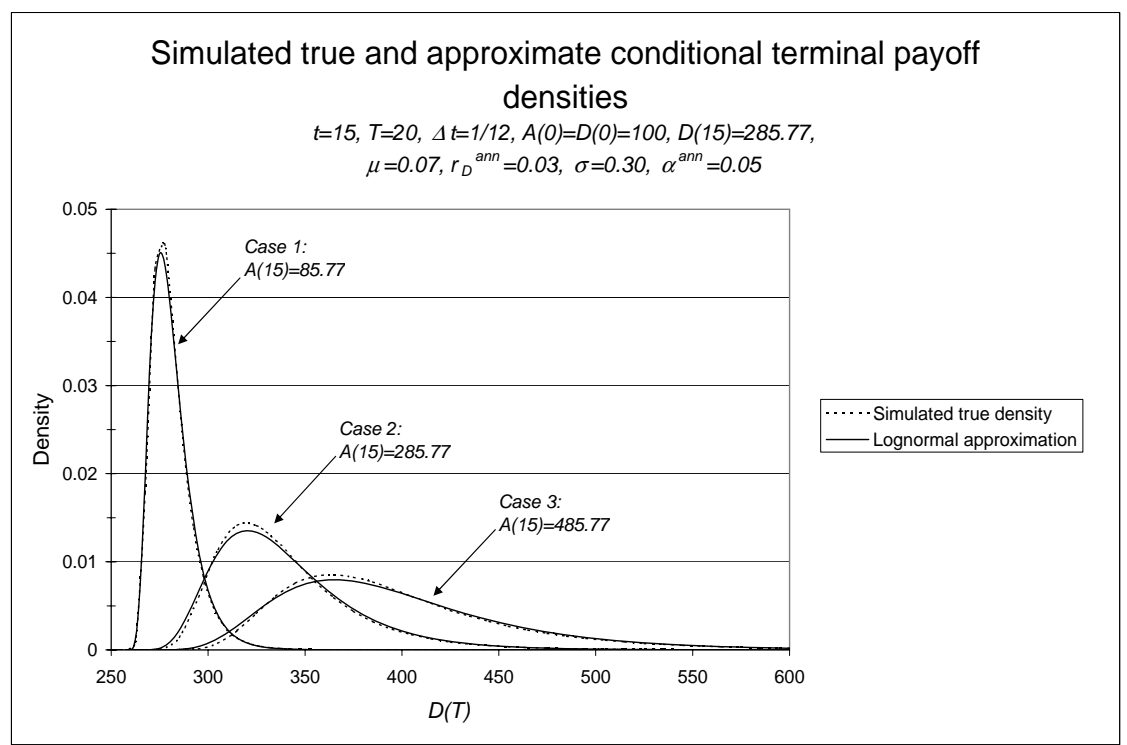

noted. Although the present policyholder account balances, the $D(15) \mathrm{s}$, are identical in the 3 cases, the payoff distributions are very different. We see that the density of the payoff distribution is centered around lower values and more spiked, the lower the market value of the underlying investment fund.

\section{Concluding remarks}

This paper has analyzed the return smoothing mechanism embedded in a new type of pension savings scheme which has been introduced recently by a Danish life insurance company.

Contrary to the widely criticized return smoothing mechanisms in traditional with-profits policies, the mechanism analyzed here is fully transparent in the sense that policyholders are fully informed about the characteristics of the underlying investment fund and about the exact way in which market returns are smoothed and credited to their personal accounts over time.

The transparency and well-definedness of this new return smoothing mechanism allowed us to establish the pension scheme's exact payoff function. This function clarifies how entering into this scheme is tantamount to holding a portfolio consisting of a certain position in the riskless asset and a position in a structured derivative with a payoff determined as an exponentially 
weighted average of market values of the underlying investment fund sampled at equally spaced time intervals over the life of the contract.

Inspired in part by the literature on the types of exotic derivatives known as Asian options, we showed how the payoff distribution of the structured derivative component of the contract could be approximated by a lognormal distribution. Since the first two true moments of the stochastic payoff of the structured derivative component could be determined analytically, we used moment matching as the criteria for identifying the specific approximating lognormal distributions.

During the course of the analysis, a range of simulation based numerical experiments were conducted to support the conclusion that the approximation technique indeed performs well.

For the holder of the type of pension savings contract described in this paper the importance and relevance of being able to analyze the contract's payoff distribution is obvious. But there are several other potentially interesting practical applications of the results established in this paper.

Firstly, our results are potentially useful in relation to the risk management of the issuing company. While the issuing company might find that the (approximating) distributions derived above are informative in their own right, it might also sensibly choose to consider its risk exposure measured as the terminal surplus $A(T)-D(T)$. By following the ideas presented in this paper one might construct accurate approximating distributions for this solvency variable in exactly the same way as we did for the payoff function. This would in turn allow for easy determination of appropriate (prudent) contract parameters. Given such a tool it would also be straightforward to conduct Value-at-Risk analysis on (cohorts of) outstanding contracts.

A second potential application of the paper's results relates to the valuation of options in connection with the pension saver's contract. For example, it is quite common that companies issue maturity guarantees along with pension savings products like the one described and analyzed in this paper. ${ }^{10}$ In the present context a guaranteed contract payoff would correspond to a riskless bond and a call option on $X_{n, N}$ being held by the pension saver. In light of the results of this paper this highly exotic and Asian-like option could be quite accurately valued by the suitably adapted version of the Black-Scholes formula (Black and Scholes (1973)) that would result from assuming the underlying variable to be lognormal. As this paper has shown, such an assumption would not be unreasonable.

An obvious extension of this work would be to investigate the performance of alternative techniques for approximating the sum of lognormal variables which appeared in the pension

\footnotetext{
${ }^{10}$ For an analysis of maturity guarantees in relation to pension savings contract see Boyle and Hardy (1997).
} 
contract payoff function studied in this paper. In this respect, the theory of comonotonicity put forward in a series of recent papers (e.g. Dhaene et al. (2002a) and Dhaene et al. (2002b)) appears most promising. For example, in a study of risk measures related to discounted sums of nonindenpent lognormal random variables Vanduffel, Hoedemakers, and Dhaene (2005) report that their comonotonic "maximal variance" lower bound approximation outperforms both the usual lognormal approximation and the approximation based on the reciprocal gamma distribution (see also Vanduffel et al. (2006)). It would be interesting to conduct a similar study for the somewhat different payoff function/contract type presented in this paper. 


\section{These appendices are supplied for the referee's convenience The paper can be published with or without them}

\section{A Proof of Lemma 1}

For ease of notation define $\omega=(1-\alpha)\left(1+r_{D}\right)$. Then for $t \in\left[t_{n}, t_{n+1}[\right.$ and $n \in\{0,1, \ldots, N-1\}$ we have

$$
\begin{aligned}
E\left\{X_{n, N} \mid \mathcal{F}_{t}\right\} & =E\left\{\alpha \sum_{i=n+1}^{N} \omega^{N-i} A\left(t_{i}\right) \mid \mathcal{F}_{t}\right\} \\
& =\alpha \sum_{i=n+1}^{N} \omega^{N-i} A(t) e^{\mu\left(t_{i}-t\right)} \\
& =\alpha A(t) e^{-\mu t} \sum_{i=n+1}^{N} \omega^{N-i} e^{\mu \Delta t \cdot i} e^{\mu \Delta t \cdot(N-N)} \\
& =\alpha A(t) e^{\mu(T-t)} \sum_{i=n+1}^{N}\left[\frac{\omega}{e^{\mu \Delta t}}\right]^{N-i} \\
& =\alpha A(t) e^{\mu(T-t)} \sum_{j=0}^{N-n-1}\left[\frac{\omega}{e^{\mu \Delta t}}\right]^{j} \\
& =\alpha A(t) e^{\mu(T-t)} \frac{1-\left[\frac{\omega}{e^{\mu \Delta t}}\right]^{N-n}}{1-\left[\frac{\omega}{e^{\mu \Delta t}}\right]}, \quad \text { for }\left[\frac{\omega}{e^{\mu \Delta t}}\right] \neq 1 .
\end{aligned}
$$

Q.E.D. 


\section{B Proof of Lemma 2}

Again we let $\omega=(1-\alpha)\left(1+r_{D}\right)$, and $t \in\left[t_{n}, t_{n+1}[\right.$ with $n \in\{0,1, \ldots, N-1\}$, and we consider

$$
\begin{aligned}
E\left\{X_{n, N}^{2} \mid \mathcal{F}_{t}\right\} & =E\left\{\left(\alpha \sum_{i=n+1}^{N}(1-\alpha)^{N-i}\left(1+r_{D}\right)^{N-i} A\left(t_{i}\right)\right)^{2} \mid \mathcal{F}_{t}\right\} \\
& =E\left\{\alpha^{2} \sum_{i=n+1}^{N} \sum_{j=n+1}^{N} \omega^{N-i} \omega^{N-j} A\left(t_{i}\right) A\left(t_{j}\right) \mid \mathcal{F}_{t}\right\} \\
& =\alpha^{2} \sum_{i=n+1}^{N} \sum_{j=n+1}^{N} \omega^{N-i} \omega^{N-j} E\left\{A\left(t_{i}\right) A\left(t_{j}\right) \mid \mathcal{F}_{t}\right\} .
\end{aligned}
$$

Using (5) and standard results regarding the properties of Brownian motion, the expectation in (B.1) can be evaluated as

$$
E\left\{A\left(t_{i}\right) A\left(t_{j}\right) \mid \mathcal{F}_{t}\right\}=A(t)^{2} e^{\mu\left(t_{i}-t+t_{j}-t\right)+\sigma^{2} \min \left(t_{i}-t, t_{j}-t\right)},
$$

and (B.1) can thus be continued as follows

$$
\begin{aligned}
= & \alpha^{2} A(t)^{2} \sum_{i=n+1}^{N} \sum_{j=n+1}^{N} \omega^{N-i} \omega^{N-j} e^{\mu\left(t_{i}-t+t_{j}-t\right)+\sigma^{2} \min \left(t_{i}-t, t_{j}-t\right)} \\
= & \alpha^{2} A(t)^{2} \times \\
& {\left[\sum_{i=n+1}^{N} \sum_{j=n+1}^{i-1} \omega^{N-i} \omega^{N-j} e^{\mu\left(t_{i}-t+t_{j}-t\right)+\sigma^{2}\left(t_{j}-t\right)}+\sum_{i=n+1}^{N} \sum_{j=i}^{N} \omega^{N-i} \omega^{N-j} e^{\mu\left(t_{i}-t+t_{j}-t\right)+\sigma^{2}\left(t_{i}-t\right)}\right] } \\
= & \alpha^{2} A(t)^{2} e^{-\left(2 \mu+\sigma^{2}\right) t} \times \\
& {\left[\sum_{i=n+1}^{N} \omega^{N-i} e^{\mu \Delta t \cdot i} \sum_{j=n+1}^{i-1} \omega^{N-j} e^{\left(\mu+\sigma^{2}\right) \Delta t \cdot j}+\sum_{i=n+1}^{N} \omega^{N-i} e^{\left(\mu+\sigma^{2}\right) \Delta t \cdot i} \sum_{j=i}^{N} \omega^{N-j} e^{\mu \Delta t \cdot j}\right] }
\end{aligned}
$$

For ease of notation we now define $\Gamma=\frac{\omega}{e^{\mu \Delta t}}$ and $\Lambda=\frac{\omega}{e^{\left(\mu+\sigma^{2}\right) \Delta t}}$, and consider next the first inner sum of (B.2). Assuming $\Gamma \neq 1, \Lambda \neq 1$, and $\Gamma \Lambda \neq 1$, we get

$$
\begin{aligned}
\sum_{j=n+1}^{i-1} \omega^{N-j} e^{\left(\mu+\sigma^{2}\right) \Delta t \cdot j} & =\sum_{j=N-i+1}^{N-n-1} \omega^{j} e^{\left(\mu+\sigma^{2}\right) \Delta t \cdot(N-j)} \\
& =e^{\left(\mu+\sigma^{2}\right) T} \sum_{j=N-i+1}^{N-n-1} \Lambda^{j} \\
& =e^{\left(\mu+\sigma^{2}\right) T}\left[\frac{\Lambda^{N-n}-\Lambda^{N-i+1}}{\Lambda-1}\right] .
\end{aligned}
$$


The first double sum component of (B.2) is thus

$$
\begin{aligned}
& \sum_{i=n+1}^{N} \omega^{N-i} e^{\mu \Delta t \cdot i} e^{\left(\mu+\sigma^{2}\right) T}\left[\frac{\Lambda^{N-n}-\Lambda^{N-i+1}}{\Lambda-1}\right] \\
= & e^{\left(\mu+\sigma^{2}\right) T} \sum_{i=0}^{N-n-1} \omega^{i} e^{\mu \Delta t \cdot(N-i)\left[\frac{\Lambda^{N-n}-\Lambda^{i+1}}{\Lambda-1}\right]} \\
= & e^{\left(2 \mu+\sigma^{2}\right) T} \sum_{i=0}^{N-n-1} \Gamma^{i}\left[\frac{\Lambda^{N-n}-\Lambda^{i+1}}{\Lambda-1}\right] \\
= & e^{\left(2 \mu+\sigma^{2}\right) T}\left[\left(\frac{\Lambda^{N-n}}{\Lambda-1}\right)\left(\frac{\Gamma^{N-n}-1}{\Gamma-1}\right)-\left(\frac{\Lambda}{\Lambda-1}\right) \sum_{i=0}^{N-n-1}(\Gamma \Lambda)^{i}\right] \\
= & e^{\left(2 \mu+\sigma^{2}\right) T}\left[\left(\frac{\Lambda^{N-n}}{\Lambda-1}\right)\left(\frac{\Gamma^{N-n}-1}{\Gamma-1}\right)-\left(\frac{\Lambda}{\Lambda-1}\right)\left(\frac{(\Gamma \Lambda)^{N-n}-1}{\Gamma \Lambda-1}\right)\right] .
\end{aligned}
$$

Now, similar manipulation of the second inner sum of (B.2) yields

$$
\sum_{j=i}^{N} \omega^{N-j} e^{\mu \Delta t \cdot j}=\sum_{j=0}^{N-i} \omega^{j} e^{\mu \Delta t \cdot(N-j)}=e^{\mu T} \sum_{j=0}^{N-i} \Gamma^{j}=e^{\mu T}\left[\frac{\Gamma^{N-i+1}-1}{\Gamma-1}\right] .
$$

The second double sum displayed in (B.2) thus becomes

$$
\begin{aligned}
& \sum_{i=n+1}^{N} \omega^{N-i} e^{\left(\mu+\sigma^{2}\right) \Delta t \cdot i} e^{\mu T}\left[\frac{\Gamma^{N-i+1}-1}{\Gamma-1}\right] \\
= & e^{\mu T}\left[\frac{1}{\Gamma-1} \sum_{i=0}^{N-n-1} \omega^{i} e^{\left(\mu+\sigma^{2}\right) \Delta t \cdot(N-i)}\left(\Gamma^{i+1}-1\right)\right] \\
= & e^{\left(2 \mu+\sigma^{2}\right) T}\left[\frac{1}{\Gamma-1}\left(\Gamma \sum_{i=0}^{N-n-1}(\Gamma \Lambda)^{i}-\sum_{i=0}^{N-n-1} \Lambda^{i}\right)\right] \\
= & e^{\left(2 \mu+\sigma^{2}\right) T}\left[\left(\frac{\Gamma}{\Gamma-1}\right)\left(\frac{(\Gamma \Lambda)^{N-n}-1}{\Gamma \Lambda-1}\right)-\left(\frac{1}{\Gamma-1}\right)\left(\frac{\Lambda^{N-n}-1}{\Lambda-1}\right)\right] .
\end{aligned}
$$

A final collection of terms yields the desired result

$$
\begin{aligned}
E\left\{X_{n, N}^{2} \mid \mathcal{F}_{t}\right\}= & \alpha^{2} A(t)^{2} e^{\left(2 \mu+\sigma^{2}\right)(T-t)} \times \\
& {\left[\left(\frac{\Lambda^{N-n}}{\Lambda-1}\right)\left(\frac{\Gamma^{N-n}-1}{\Gamma-1}\right)-\left(\frac{\Lambda}{\Lambda-1}\right)\left(\frac{(\Gamma \Lambda)^{N-n}-1}{\Gamma \Lambda-1}\right)\right.} \\
& \left.+\left(\frac{\Gamma}{\Gamma-1}\right)\left(\frac{\left.(\Gamma \Lambda)^{N-n}-1\right)}{\Gamma \Lambda-1}\right)-\left(\frac{1}{\Gamma-1}\right)\left(\frac{\Lambda^{N-n}-1}{\Lambda-1}\right)\right] .
\end{aligned}
$$

Q.E.D. 


\section{References}

Black, F. and M. Scholes (1973): “The Pricing of Options and Corporate Liabilities,” Journal of Political Economy, 81(3):637-654.

Boyle, P. P. and M. R. Hardy (1997): “Reserving for Maturity Guarantees: Two Approaches,” Insurance: Mathematics and Economics, 21:113-127.

Brennan, M. J. (1993): “Aspects of Insurance, Intermediation and Finance," The Geneva Papers on Risk and Insurance Theory, 18(1):7-30.

Dhaene, J., M. Denuit, M. Goovaerts, R. Kaas, and D. Vyncke (2002a): "The Concept of Comonotonicity in Actuarial Science and Finance: Theory," Insurance: Mathematics and Economics, 31(1):3-33.

(2002b): “The Concept of Comonotonicity in Actuarial Science and Finance: Applications,” Insurance: Mathematics and Economics, 31(2):133-161.

Dufresne, D. (1990): “The Distribution of a Perpetuity with Applications to Risk Theory and Pension Funding,” Scandinavian Actuarial Journal, pages 39-79.

(2004): "The Log-normal Approximation in Financial and Other Computations," Advances in Applied Probability, 36(3):747-773.

Grosen, A. and P. L. Jørgensen (2002): “The Bonus-crediting Mechanism of Danish Pension and Life Insurance Companies: An Empirical Analysis,” Journal of Pension Economics and Finance, 1(3):249-268.

Guillen, M., P. L. Jørgensen, and J. P. Nielsen (2006): "Return Smoothing Mechanisms in Life and Pension Insurance: Path-dependent Contingent Claims,” Insurance: Mathematics and Economics, 38(2):229-252.

Hansen, A. and P. L. Jørgensen (2000): “Analytical Valuation of American-style Asian Options,” Management Science, 46(8):1116-1136.

Levy, E. (1992): “Pricing European Average Rate Currency Options,” Journal of International Money and Finance, 11:474-491.

Milevsky, M. A. and S. E. Posner (1998): "Asian Options, The Sum of Lognormals and the Reciprocal Gamma Distribution,” Journal of Financial and Quantitative Analysis, 33(3):409422.

Vanduffel, S., X. Chen, J. Dhaene, M. Goovaerts, L. Henrard, and R. Kaas (2006): “A Note on Optimal Lower Bound Approximations for Risk Measures of Sums of Lognormals,” Working Paper, Actuarial Science Research Group, Katholieke Universiteit Leuven.

Vanduffel, S., T. Hoedemakers, and J. Dhaene (2005): “Comparing Approximations for Risk Measures of Sums of Nonindependent Lognormal Random Variables,” North American Actuarial Journal, 9(4):71-82. 
Figure 5: Case 1. Short maturity, low volatility, strong smoothing.
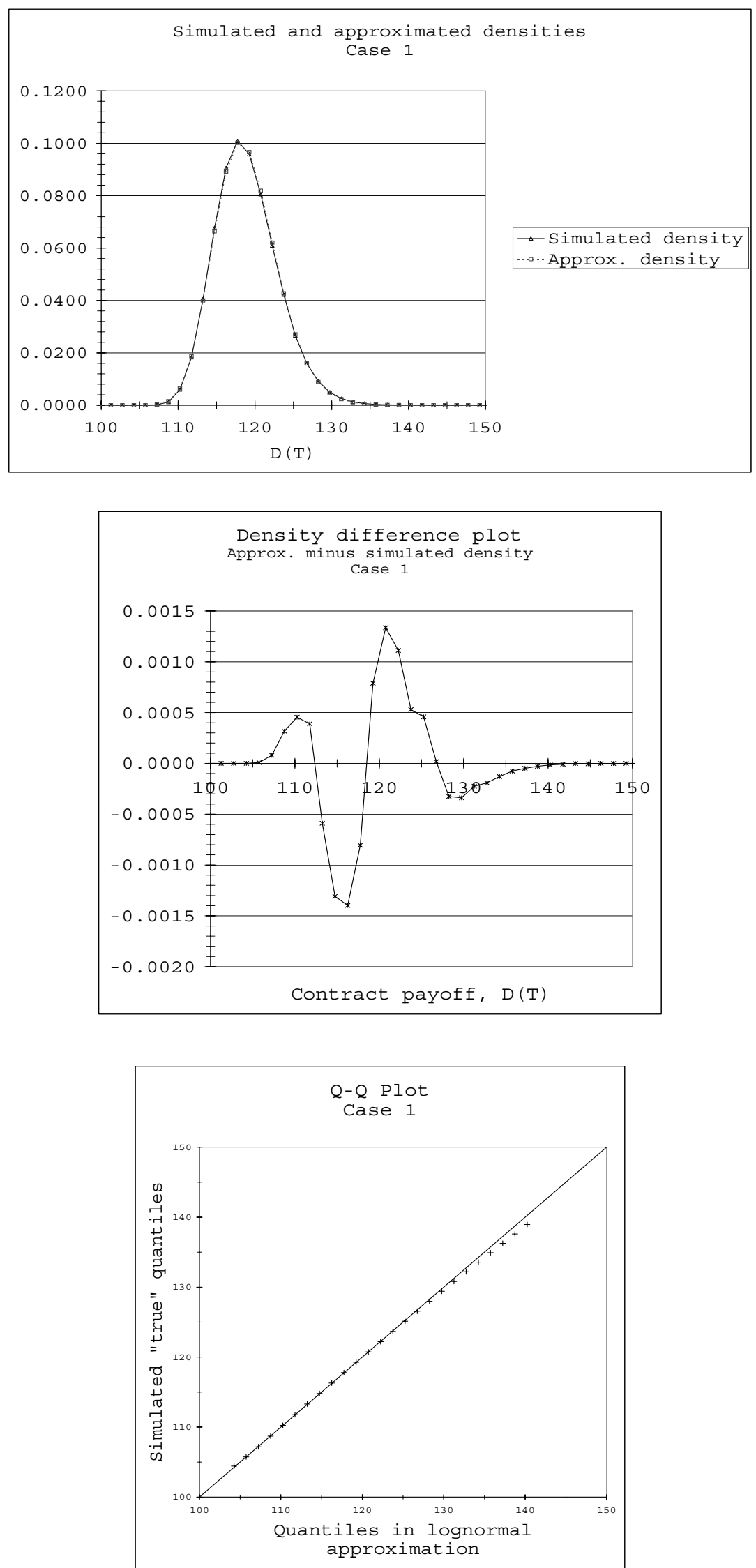
Figure 6: Case 2. Short maturity, low volatility, weak smoothing.
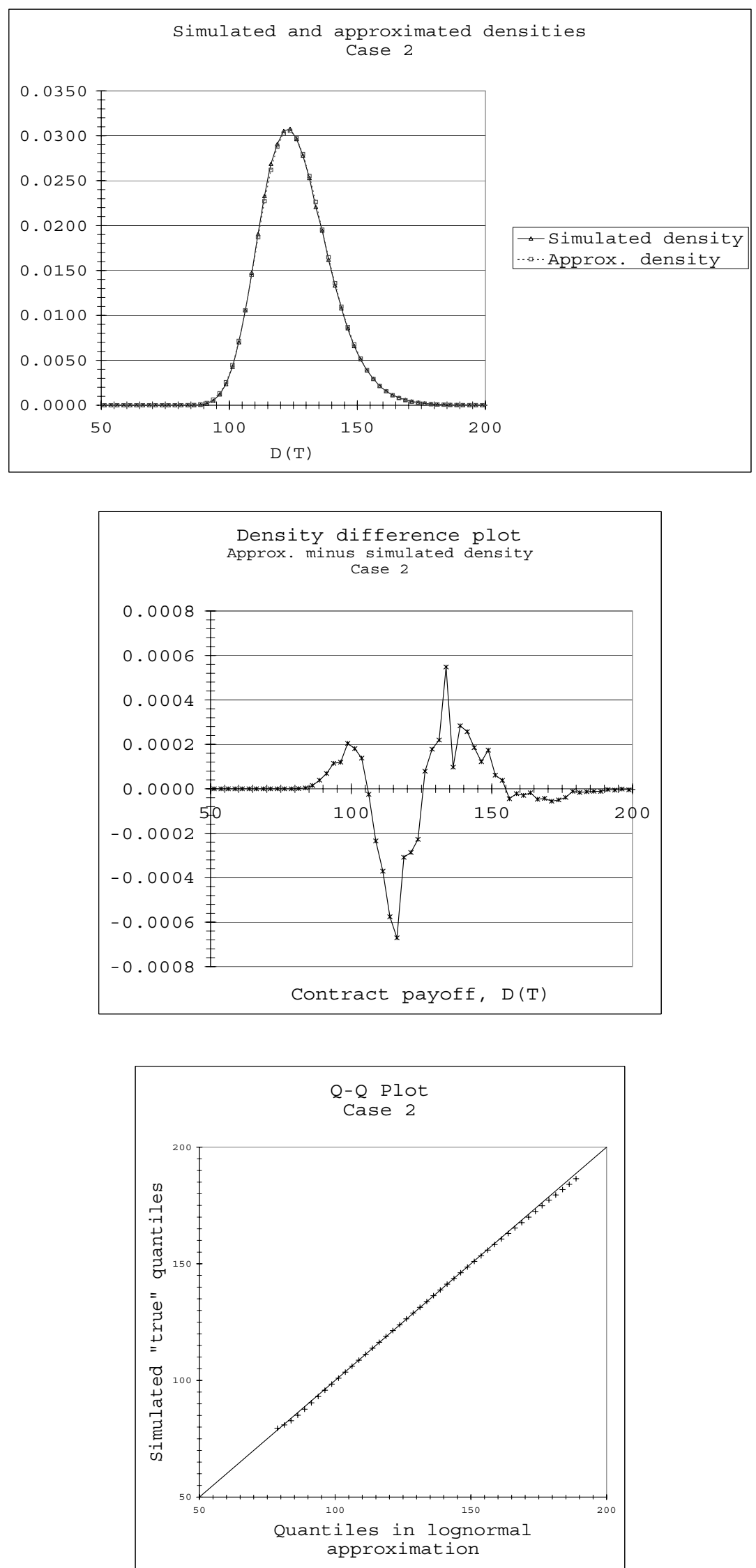
Figure 7: Case 3. Short maturity, high volatility, strong smoothing.
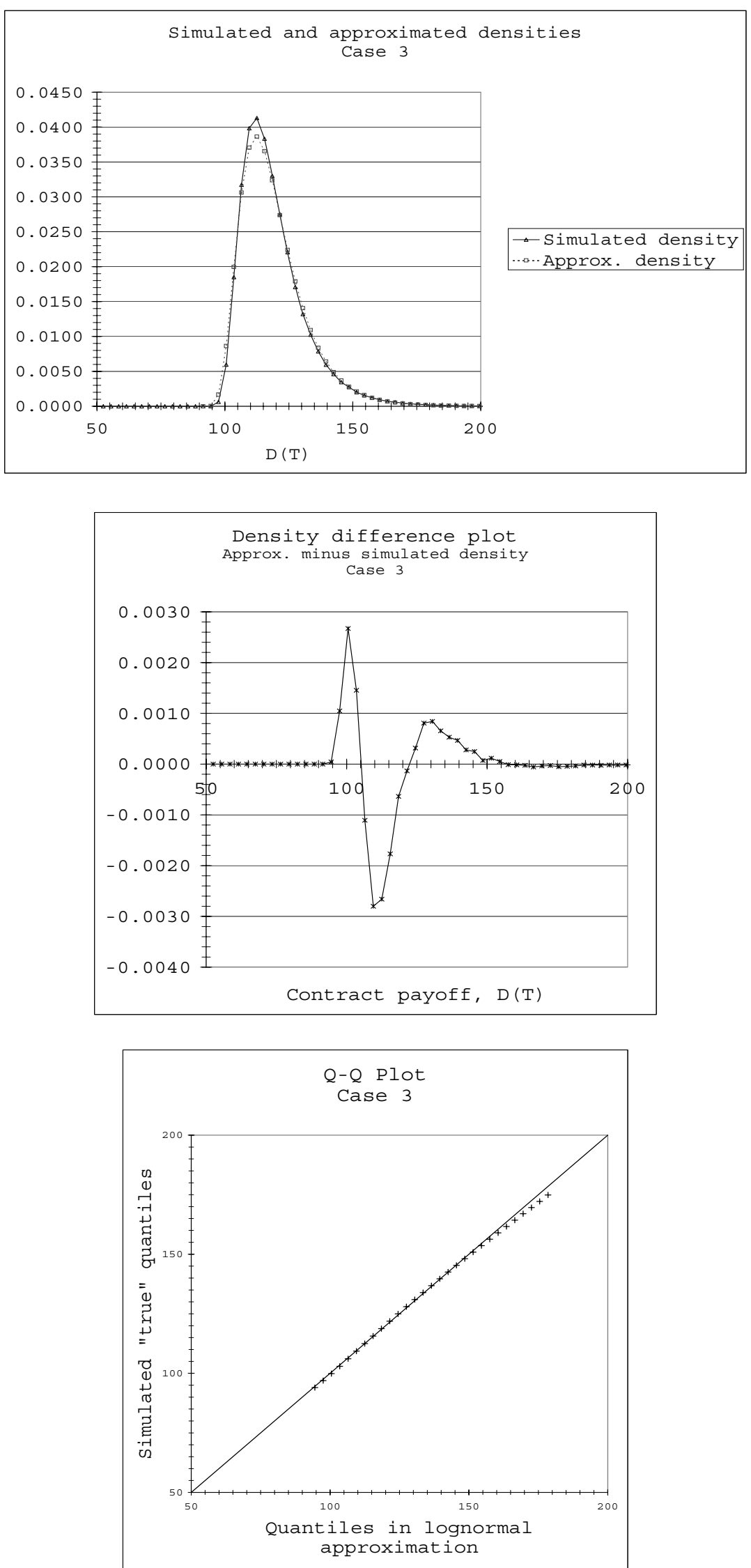
Figure 8: Case 4. Short maturity, high volatility, weak smoothing.
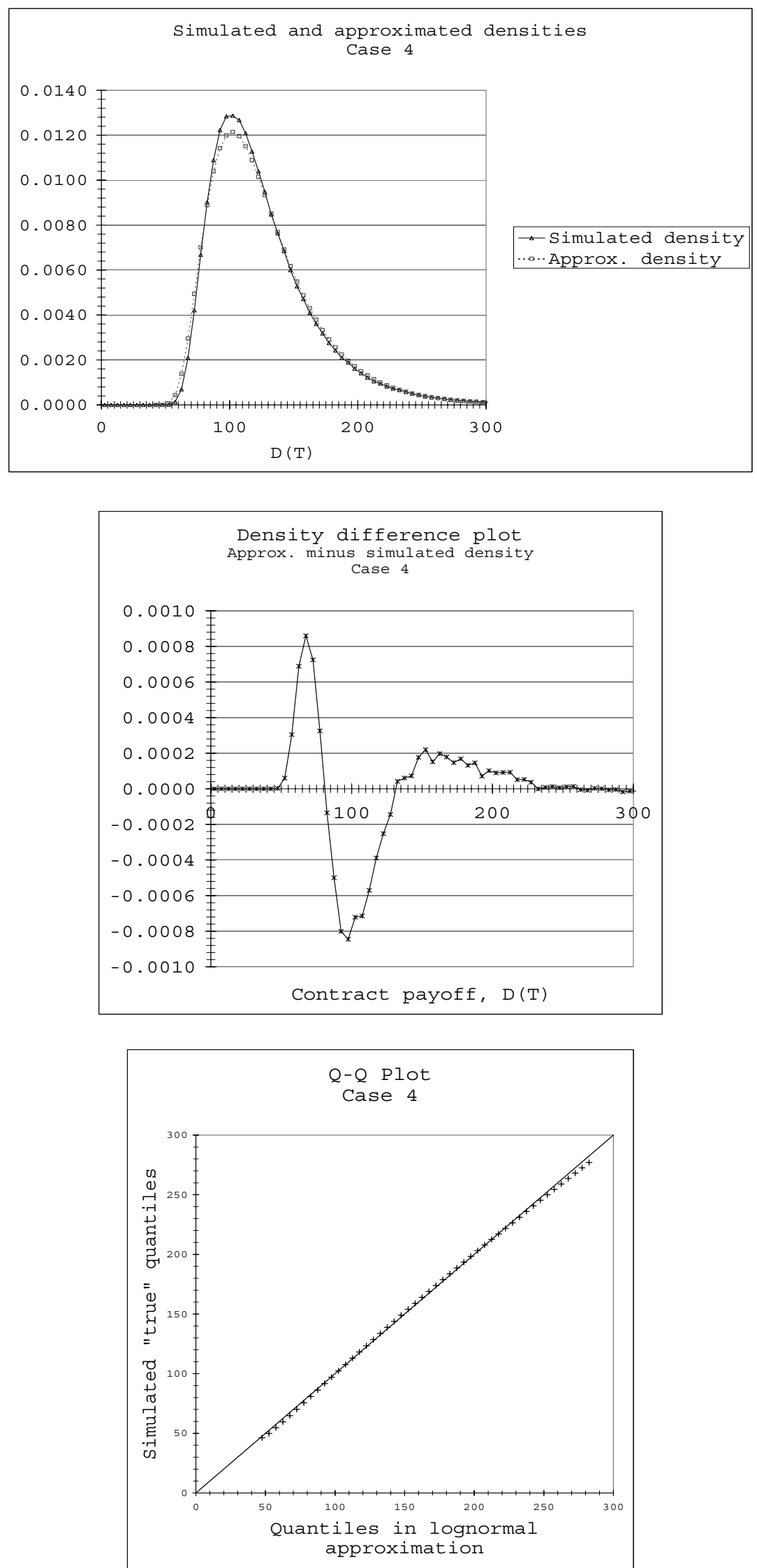
Figure 9: Case 5. Long maturity, low volatility, strong smoothing.
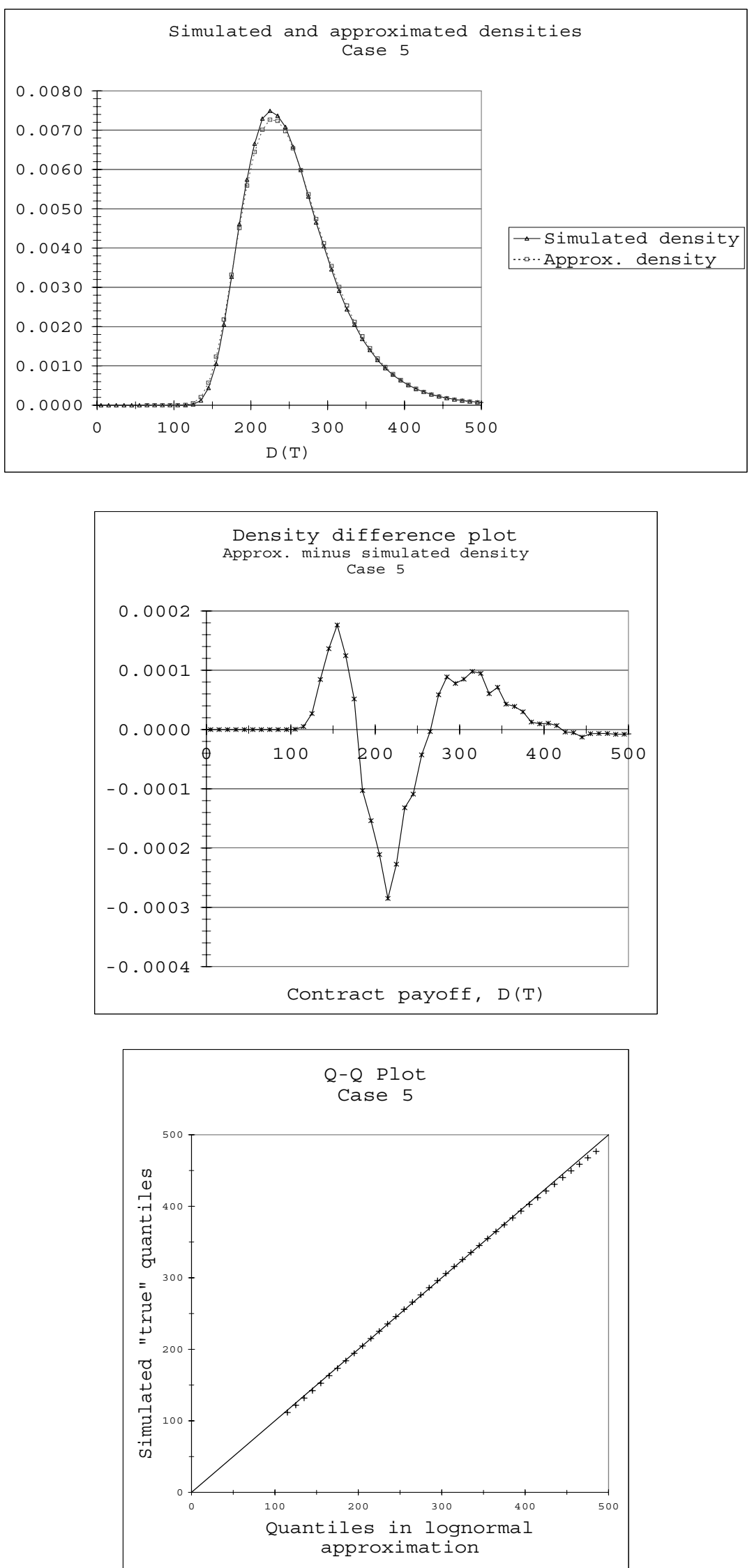
Figure 10: Case 6. Long maturity, low volatility, weak smoothing.
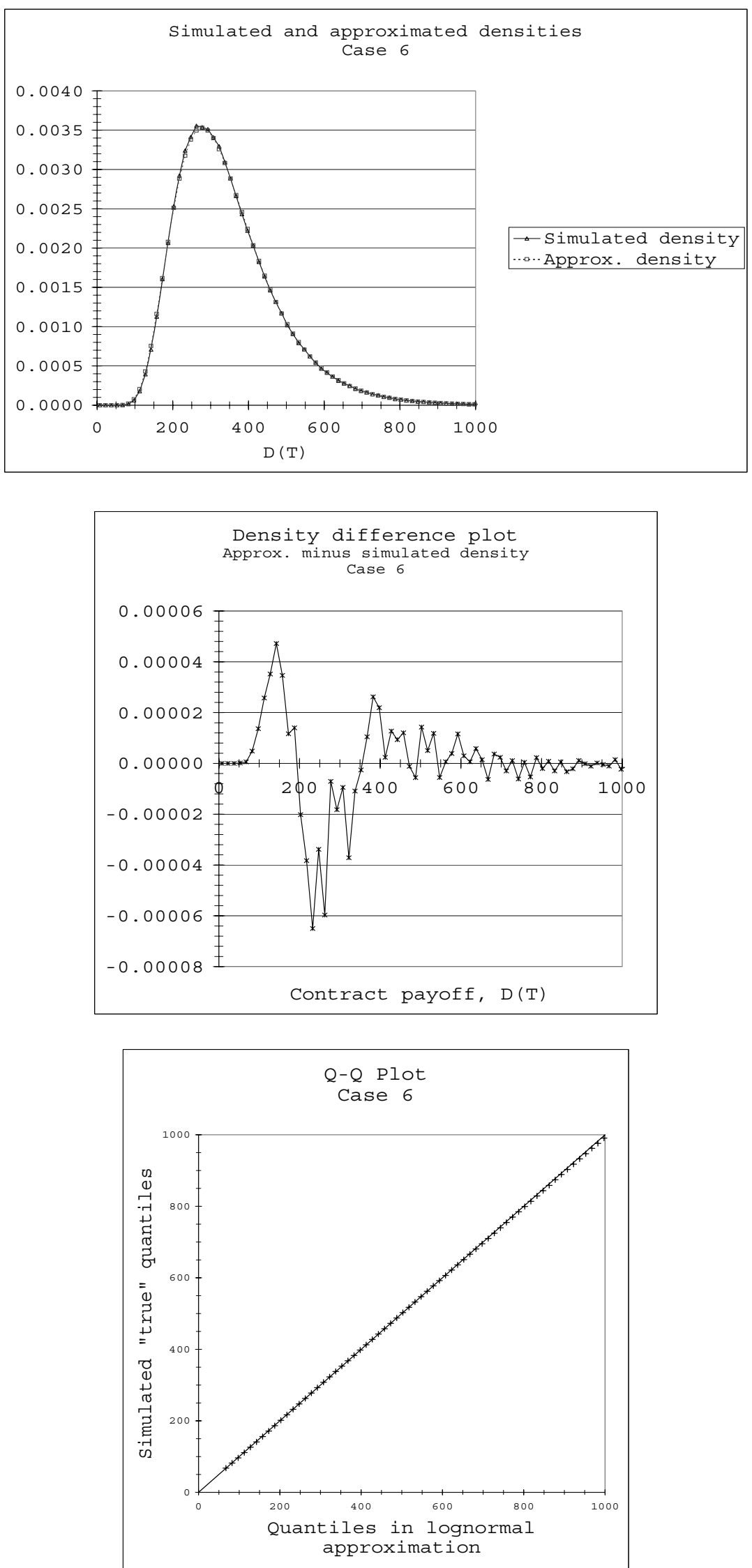
Figure 11: Case 7. Long maturity, high volatility, strong smoothing.
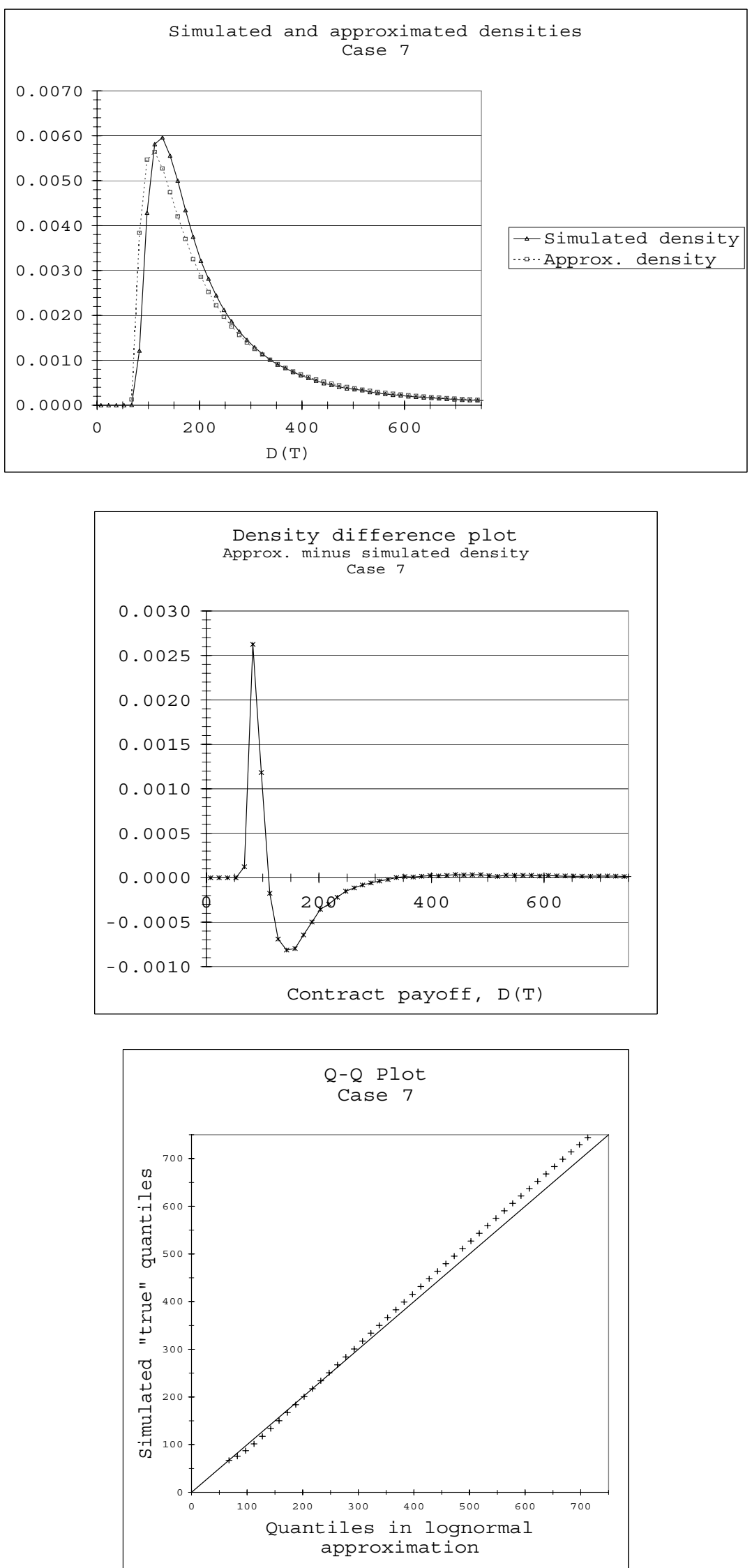
Figure 12: Case 8. Long maturity, high volatility, weak smoothing.
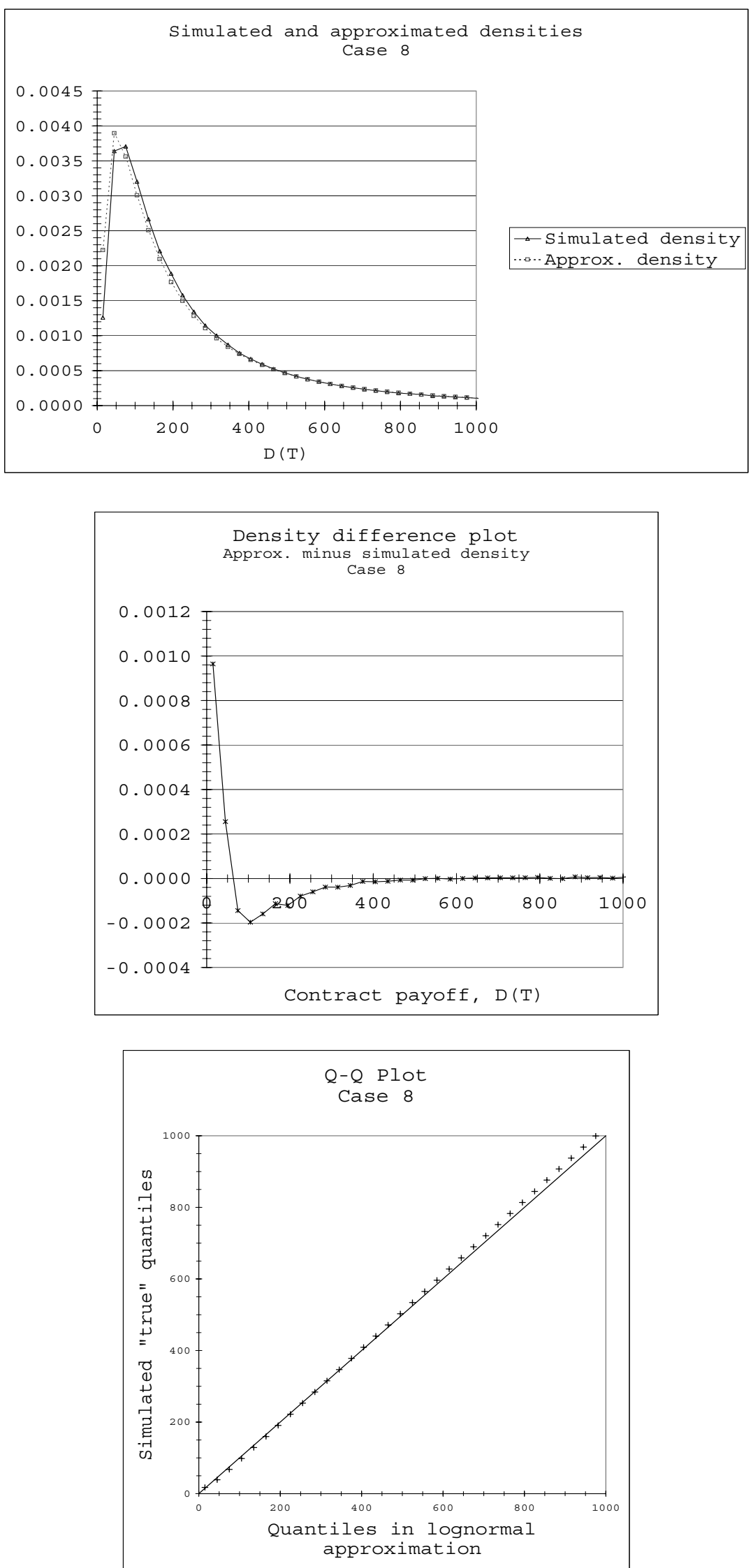


\section{Working Papers from Finance Research Group}

F-2006-09 Peter Løchte Jørgensen: Lognormal Approximation of Complex Pathdependent Pension Scheme Payoffs.

F-2006-08 Peter Løchte Jørgensen: Traffic Light Options.

F-2006-07 David C. Porter, Carsten Tanggaard, Daniel G. Weaver \& Wei Yu: Dispersed Trading and the Prevention of Market Failure: The Case of the Copenhagen Stock Exhange.

F-2006-06 Amber Anand, Carsten Tanggaard \& Daniel G. Weaver: Paying for Market Quality.

F-2006-05 Anne-Sofie Reng Rasmussen: How well do financial and macroeconomic variables predict stock returns: Time-series and cross-sectional evidence.

F-2006-04 Anne-Sofie Reng Rasmussen: Improving the asset pricing ability of the Consumption-Capital Asset Pricing Model.

F-2006-03 Jan Bartholdy, Dennis Olson \& Paula Peare: Conducting event studies on a small stock exchange.

F-2006-02 Jan Bartholdy \& Cesário Mateus: Debt and Taxes: Evidence from bankfinanced unlisted firms.

F-2006-01 Esben P. Høg \& Per H. Frederiksen: The Fractional Ornstein-Uhlenbeck Process: Term Structure Theory and Application.

F-2005-05 Charlotte Christiansen \& Angelo Ranaldo: Realized bond-stock correlation: macroeconomic announcement effects.

F-2005-04 Søren Willemann: GSE funding advantages and mortgagor benefits:

Answers from asset pricing.

F-2005-03 Charlotte Christiansen: Level-ARCH short rate models with regime switching: Bivariate modeling of US and European short rates.

F-2005-02 Charlotte Christiansen, Juanna Schröter Joensen and Jesper Rangvid: Do more economists hold stocks?

F-2005-01 Michael Christensen: Danish mutual fund performance - selectivity, market timing and persistence.

F-2004-01 Charlotte Christiansen: Decomposing European bond and equity volatility. 
ISBN 87-7882-185-1

Department of Business Studies

Aarhus School of Business

Fuglesangs Allé 4

DK-8210 Aarhus V - Denmark

Tel. +4589486688

Fax +4586150188

www.asb.dk 